\title{
Onset and Dynamics of Vortex-Antivortex Pairs in Polariton Optical Parametric Oscillator Superfluids
}

\author{
G. Tosi, ${ }^{1}$ F. M. Marchetti, ${ }^{2, *}$ D. Sanvitto, ${ }^{3}$ C. Antón, ${ }^{1}$ M. H. Szymańska, ${ }^{4, \dagger}$ \\ A. Berceanu, ${ }^{2}$ C. Tejedor, ${ }^{2}$ L. Marrucci, ${ }^{5}$ A. Lemaître, ${ }^{6}$ J. Bloch, ${ }^{6}$ and L. Viña ${ }^{1}$ \\ ${ }^{1}$ Física de Materiales, Universidad Autónoma de Madrid, Madrid 28049, Spain \\ ${ }^{2}$ Física Teórica de la Materia Condensada, Universidad Autónoma de Madrid, Madrid 28049, Spain \\ ${ }^{3}$ NNL, Istituto Nanoscienze - CNR, Via Arnesano, 73100 Lecce, Italy \\ ${ }^{4}$ Department of Physics, University of Warwick, Coventry, CV4 7AL, United Kingdom \\ ${ }^{5}$ Dipartimento di Scienze Fisiche, Università di Napoli Federico II and CNR-SPIN, Napoli, Italy \\ ${ }^{6}$ LPN/CNRS, Route de Nozay, 91460, Marcoussis, France \\ (Received 17 March 2011; revised manuscript received 27 May 2011; published 12 July 2011)
}

\begin{abstract}
We study, both theoretically and experimentally, the occurrence of topological defects in polariton superfluids in the optical parametric oscillator (OPO) regime. We explain in terms of local supercurrents the deterministic behavior of both the onset and dynamics of vortex-antivortex pairs generated by perturbing the system with a pulsed probe. Using a generalized Gross-Pitaevskii equation, including photonic disorder, pumping and decay, we elucidate the reason why topological defects form in couples and can be detected by direct visualizations in multishot OPO experiments.
\end{abstract}

DOI: 10.1103/PhysRevLett.107.036401

PACS numbers: 71.36.+c, 42.65.Yj, 47.32.C-

Quantum vortices are topological defects occurring in macroscopically coherent systems. Their existence was first predicted in superfluids [1,2], and later in coherent waves [3]. Nowadays, quantum vortices have been the subject of extensive research across several areas of physics and have been observed in type-II superconductors, ${ }^{4} \mathrm{He}$, ultracold atomic gases, nonlinear optics media (for a review see, e.g., $[4,5]$ ) and very recently microcavity polaritons [6-13]. The phase of a quantized vortex winds around its core from 0 to $2 \pi m$ (with $m$ integer), implying the vortex carries a quantized angular momentum, $\mathrm{hm}$. In contrast with the classical counterpart, quantum vortices with the same $m$ are all identical, with a size (or healing length) determined by the system nonlinear properties.

Recently, the study of quantized vortices imprinted in polariton condensates using pulsed laser fields has attracted noticeable interest both experimentally [9] and theoretically [14-17], providing a diagnostics for superfluid properties of such a nonequilibrium system. In particular, resonantly pumped polaritons in the optical parametric oscillator (OPO) regime $[18,19]$ have been recently shown to exhibit a new form of nonequilibrium superfluidity $[9,20]$. Here, polaritons continuously injected into the pump state, undergo coherent stimulated scattering into the signal and idler states. An additional pulsed probe can initiate a traveling decaying gain, which evolves freely from the probe constraints. By using a pulsed LaguerreGauss (LG) beam, vorticity has been shown to persist not only in absence of the rotating drive, but also longer than the gain induced by the probe, and therefore to be transferred to the OPO signal, demonstrating metastability of quantum vortices and persistence of currents $[9,15]$.

However, if the extension of the probe carrying a vortex with charge $m=+1$ is smaller than the size of the vortex- free OPO signal, continuity of the polariton wave function requires that necessarily an antivortex with charge $m=-1$ has to form at the edge of the probe (see Fig. 1). In this Letter, we demonstrate that "unintended" antivortices do appear in the signal at the edge of the imprinting vortex probe and explain, both theoretically and via experiments, the origin of the deterministic behavior of the antivortex onset and dynamics. In particular, we show where antivortices are more likely to appear in terms of the supercurrents of the imprinting probe and the ones of the underlying OPO. In addition, our study reveals that the onset of vortices in polariton superfluids does not require a LG imprinting beam, but instead vortex-antivortex (V-AV) pairs can also be generated when counterpropagating currents are imposed, similarly to what happens in normal (classical) fluids.

Crucially, via numerical simulations, we elucidate the reason why an experimental average over many shots allows detecting a vortex by direct visualization in density and phase profiles. Recently, it has been suggested by stochastic simulations [14] that vortices in nonresonantly pumped polariton condensates undergo a random motion which will hinder their direct detection, unless they are close to being pinned by the stationary disorder potential and thus follow a deterministic trajectory [8]. In the case considered here of a superfluid generated by the OPO, we can instead explain a deterministic dynamics of the V-AV pair in terms of the OPO steady state supercurrents, which determine a unique trajectory for the pair, allowing their observation in multishot measurements.

Model.-The generalized Gross-Pitaevskii equations,

$$
i \partial_{t}\left(\begin{array}{l}
\psi_{X} \\
\psi_{C}
\end{array}\right)=\left(\begin{array}{l}
0 \\
F
\end{array}\right)+\left[\hat{H}_{0}+\left(\begin{array}{cc}
g\left|\psi_{X}\right|^{2} & 0 \\
0 & V
\end{array}\right)\right]\left(\begin{array}{l}
\psi_{X} \\
\psi_{C}
\end{array}\right),
$$



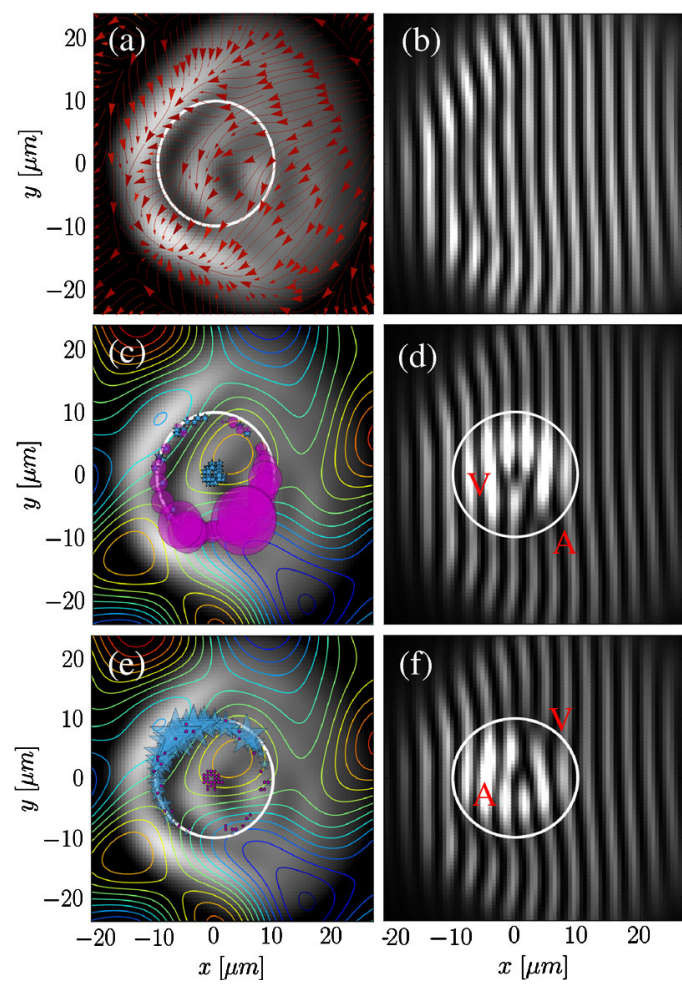

FIG. 1 (color online). Simulated profile and supercurrents of the steady state OPO signal before the arrival of the probe (a) and associated interference fringes (b). Location of antivortices [dots (c)] and vortices [stars (e)] at the arrival $(t=0 \mathrm{ps})$ of a vortex [stars (c)] or an antivortex [dots (e)] probe, for 1000 realizations of the random relative phase between pump and probe, $\Phi_{\mathrm{rdm}}$. The size of the dots in (c) [stars in (e)] is proportional to the number of times the antivortices (vortices) appear in that location. Panel (d) [(f)] shows single shot interference fringes relative to the plot in (c) [(e)]. Contour-level lines in (c) and (e) represent the photonic disorder $V(\mathbf{r})$. The white circle represents the edge of the probe.

for coupled cavity and exciton fields $\psi_{C, X}(\mathbf{r}, t)$ with pump and decay [21] model the OPO dynamics $(\hbar=1)$. The exciton-exciton interaction induces a nonlinear dynamics of lower (LP) and upper (UP) polaritons, the eigenstates of the noninteracting Hamiltonian:

$$
\hat{H}_{0}=\left(\begin{array}{cc}
\omega_{X}-i \kappa_{X} & \Omega_{R} / 2 \\
\Omega_{R} / 2 & \omega_{C}-\nabla^{2} /\left(2 m_{C}\right)-i \kappa_{C}
\end{array}\right) \text {. }
$$

The cavity (exciton) field decays with rate $\kappa_{C}\left(\kappa_{X}\right)$ and is replenished by a continuous wave (cw) laser, $F_{p}(\mathbf{r}, t)=$ $\mathcal{F}_{f_{p}, \sigma_{p}}(r) e^{i\left(\mathbf{k}_{p} \cdot \mathbf{r}-\omega_{p} t\right)}$, with a top-hat profile [22]. Above a pump strength threshold, the system is driven into the OPO regime where signal and idler states (with energies $\omega_{s, i}$ and wave vectors $\mathbf{k}_{s, i}$ ) get exponentially populated. In addition, the OPO is probed by an extra pulsed laser $F_{p b}(\mathbf{r}, t)$. As single shot measurements would give a too low signal to noise ratio, an average is performed over many pulsed experiments taken always for the same OPO conditions.
What differs at each probe arrival is the random relative phase $\Phi_{\text {rdm }}$ between pump and probe,

$$
F(\mathbf{r}, t)=F_{p}(\mathbf{r}, t)+F_{p b}(\mathbf{r}, t) e^{i \Phi_{\mathrm{rdm}}},
$$

with $\Phi_{\text {rdm }}$ uniformly distributed between 0 and $2 \pi$.

As already shown in Refs. [9,10,15], vortices with charge $m= \pm 1$ can be imprinted in the OPO signal and idler, by adding a LG pulsed probe:

$$
\begin{aligned}
F_{p b}(\mathbf{r}, t)= & f_{p b}\left|\mathbf{r}-\mathbf{r}_{p b}\right| e^{-\left|\mathbf{r}-\mathbf{r}_{p b}\right|^{2} /\left(2 \sigma_{p b}^{2}\right) e^{i m \varphi(\mathbf{r})}} \\
& \times e^{i\left(\mathbf{k}_{p b} \cdot \mathbf{r}-\omega_{p b} t\right)} e^{-\left(t-t_{p b}\right)^{2} /\left(2 \sigma_{t}^{2}\right)}
\end{aligned}
$$

where the probe momentum $\mathbf{k}_{p b}$ and energy $\omega_{p b}$ are resonant with, e.g., the OPO signal state. Here, the pulse lasts 2 ps only. The azimuthal angle $\varphi(\mathbf{r})$ winds from 0 to $2 \pi$ around the vortex core $\mathbf{r}_{p b}$. Finally, to mimic the experimental conditions, we include in (1) a static photonic disorder potential $V(\mathbf{r})$, with $\langle V(\mathbf{r})\rangle=0$ and $\left\langle V(\mathbf{r}) V\left(\mathbf{r}^{\prime}\right)\right\rangle=$ $\sigma_{d}^{2} e^{-\left|\mathbf{r}-\mathbf{r}^{\prime}\right|^{2} / 2 \ell_{d}^{2}}\left(\ell_{d} \simeq 20 \mu \mathrm{m}\right.$ and $\left.\sigma_{d} \simeq 0.1 \mathrm{meV}\right)$.

We solve numerically Eq. (1) on a $2 \mathrm{D}$ grid by using a 5th-order adaptive-step Runge-Kutta algorithm. We first find the steady state stationary conditions for OPO emission $\left(f_{p b}=0\right)$ and plot the OPO signal profile $\left|\psi_{C}^{s}(\mathbf{r}, t)\right| e^{i \phi_{C}^{s}(\mathbf{r}, t)}$ by, e.g., filtering in a cone around the signal momentum. In addition to the spatial profile, $\left|\psi_{C}^{s}(\mathbf{r}, t)\right|$, we also evaluate the supercurrents $\nabla \phi_{C}^{s}(\mathbf{r}, t)$ see Fig. 1(a). Note that the presence of the photonic disorder does not change qualitatively our results. Its role is to break the $y \mapsto-y$ symmetry left by the pump with $\mathbf{k}_{p}=$ $\left(k_{p x}, 0\right)$ and to change the supercurrents accordingly. Further, we simulate the dynamics following the arrival of a vortex probe (4) at $t=0 \mathrm{ps}$ for 1000 realizations of $\Phi_{\mathrm{rdm}}$ and then average $\left\langle\left|\psi_{C}^{s}(\mathbf{r}, t)\right| e^{i \phi_{C}^{s}(\mathbf{r}, t)}\right\rangle_{\Phi_{\mathrm{rdm}}}$.

Experimental setup.-The sample studied is a $\lambda / 2$ AlAs microcavity with a single GaAs quantum well placed at the antinode of the mirror-confined cavity field, giving a Rabi splitting of $4.4 \mathrm{meV}$-for details on the sample see Ref. [23]. Maintaining the sample at $10 \mathrm{~K}$, a cw Ti: Sapphire laser, $F_{p}(\mathbf{r}, t)$, resonantly pumps polaritons at $1.5283 \mathrm{eV}$ and $k_{p x}=1.4 \mu \mathrm{m}^{-1}$. Above a threshold, the system enters the steady state OPO regime. For a typical pump power of $450 \mathrm{~mW}$, the signal emits at $1.5268 \mathrm{eV}$, $1 \mathrm{meV}$ blueshifted from the LP bare dispersion. We filter the emission in $k$ space around the signal momentum. A streak camera follows the evolution of the signal after the arrival of a 2 ps-long probe pulse from a second Ti: Sapphire laser, $F_{p b}(\mathbf{r}, t)$, resonant with the OPO signal (typical power $3 \mu \mathrm{W}$ ), and an average over millions of shots is performed. The probe LG profile is generated by shining a Gaussian beam through a hologram with a forklike dislocation on its fringe pattern. Interference images between the signal and an expanded, constant phase, region of the signal are obtained in a Mach-Zehnder interferometer. 


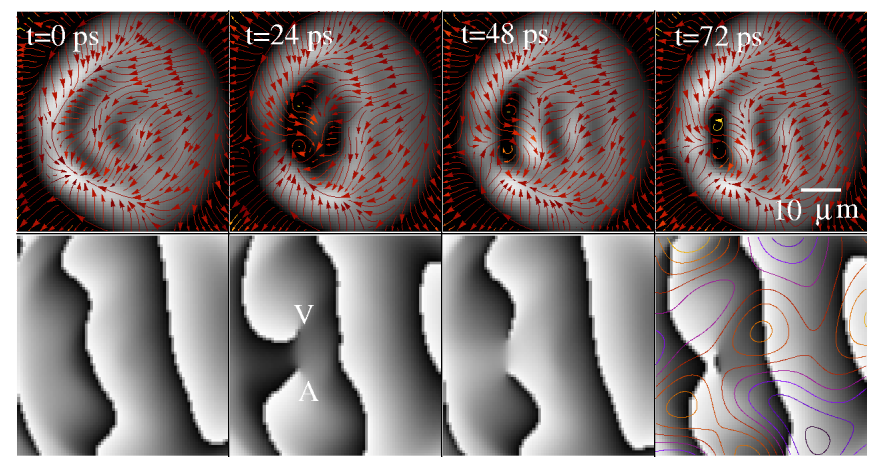

FIG. 2 (color online). Simulated time evolution of signal after the arrival of the vortex probe, averaged over 1000 realizations of the random phase $\Phi_{\mathrm{rdm}},\left\langle\psi_{C}^{s}(\mathbf{r}, t)\right\rangle_{\Phi_{\mathrm{rdm}}}$-spatial profile (top) and phase (bottom). Contour-level lines in the last panel represent the photonic disorder.

Results.- We discuss here the results obtained in the numerical simulations and later in the experiments. In both cases, we choose OPO conditions such to give a vortex-free signal [see Figs. 1(a) and 1(b)]. Nevertheless, the simultaneous presence of pump, signal, and idler emitting at different momenta, as well as the photonic disorder, implies that the OPO steady state is characterized by currents carrying polaritons from gain- to loss-dominated regions. In Fig. 1(a), the signal currents have a dominant component pointing leftwards and an equilibrium position where all currents point inwards at around $(-8,-14) \mu \mathrm{m}$.

In single shot simulations of Figs. 1(d) and 1(f) (one realization of the phase $\Phi_{\mathrm{rdm}}$ ), we find that if the probe is positioned well inside the OPO signal [e.g., $\mathbf{r}_{p b}=$ $(0,0) \mu \mathrm{m}$ ], then the imprinting of a vortex $m=+1$ (antivortex $m=-1)$ at $t=0 \mathrm{ps}$ forces the system to generate, at the same time, an antivortex $m=-1$ (vortex $m=+1$ ) at the edge of the probe. This is a consequence of the continuity of the polariton wave functions: If the signal OPO phase is homogeneous and vortex-free before the arrival of the probe, then imposing a topological defect, i.e., a branch cut, on the signal phase at the probe core, requires the branch cut to terminate where the phase is not imposed by the probe any longer and has to continuously connect to the freely chosen OPO signal phase, i.e., at the edge of the probe. Note that OPO parametric scattering processes constrain the sum of signal and idler phases to the phase of the laser pump by $2 \phi_{p}=\phi_{s}+\phi_{i}$. Thus, at the same positions where the V-AV pair appears in the signal, an AV-V pair appears in the idler, so that locally the phase constraint described above is satisfied. This agrees with the experiments in [10], though there only a single $\mathrm{V}$ $(\mathrm{AV})$ in the signal (idler) could be detected, because the signal size was comparable to the probe one.

Different relative phases $\Phi_{\text {rdm }}$ cause the antivortex (vortex) to appear in different locations around the vortex (antivortex) probe. However, in 1000 realizations of the random phase uniformly distributed between 0 and $2 \pi$, we observe that the antivortices (vortices) are more likely to appear on positions where the current of the steady state OPO signal before the probe arrival and the probe current are opposite. For example, for the $m=+1(m=-1)$ probe of Fig. 1(c) [Fig. 1(e)], the current constantly winds anticlockwise (clockwise); therefore, compared with the signal current of Fig. 1(a), the two are antiparallel in the bottom right (top left) region on the probe edge, region where it is very likely that an antivortex (vortex) is formed. Note also that the onset of antivortices (vortices) privileges regions where the steady OPO signal has a minimal intensity. Finally, wave function continuity arguments allow the formation of additional $\mathrm{V}-\mathrm{AV}$ pairs on the probe edge, but are however rare events.

By averaging the 1000 images obtained at the probe arrival ( $t=0 \mathrm{ps})$, e.g., in Fig. 1(c), neither the imprinted
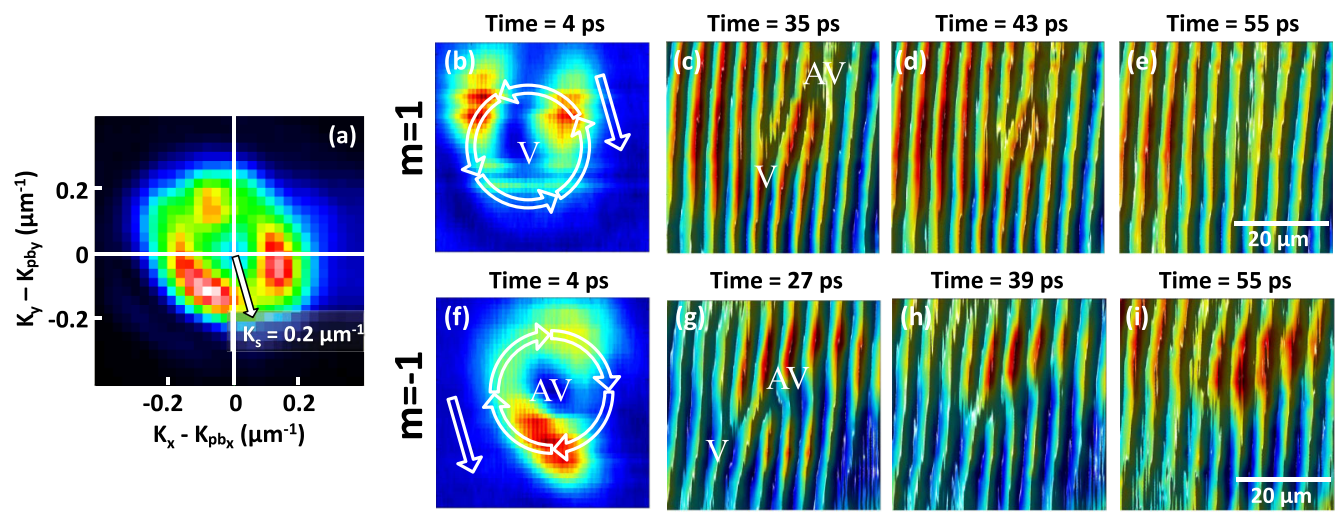

FIG. 3 (color online). Measured momentum distribution of the vortex probe vs $\mathbf{k}-\mathbf{k}_{p b}$ (a). The arrow indicates the signal momentum $\mathbf{k}_{s}-\mathbf{k}_{p b}$. Panel (b) [(f)] shows the real space emission of the signal 4 ps after the $m=1(m=-1)$ LG probe arrival. Panels (c)-(e) [panels (g)-(i)] compile, through the interference patterns, the time evolution of an imprinted $m=1$ vortex $(m=-1$ antivortex) and its associated $m=-1$ antivortex ( $m=1$ vortex). The straight arrow in (b),(f) represents the signal current direction in the probe reference frame, while the probe current winds anticlockwise for $m=1$ (clockwise for $m=-1$ ). The "unintended" antivortex (vortex) appears in (c) $[(\mathrm{g})]$ at the edge of the probe where the signal and probe currents are antiparallel. V-AV pair dynamics can be followed in both cases for about $30 \mathrm{ps}$, after which they annihilate. 
vortex nor the antivortex can be detected (see first panel of Fig. 2): Both phase singularities are washed away by averaging the differently positioned branch cuts. However, the steady state signal currents push the $\mathrm{V}$ and $\mathrm{AV}$, initially positioned in different locations, towards the same equilibrium position where all currents point inwards. Thus, while at $t=0 \mathrm{ps,}$ on average there is no $\mathrm{V}-\mathrm{AV}$ pair, after $\sim 10 \mathrm{ps,}$ both $\mathrm{V}$ and $\mathrm{AV}$ appear and last $\sim 75$ ps (see Fig. 2), till they eventually annihilate.

The theoretical predictions are borne out by the experimental observations. In order to confirm the role played by the relative currents between the probe and the OPO signal on the appearance of the "unintended" antivortex (vortex), we inject the vortex (antivortex) probe with a finite momentum with respect to that of the signal. In Fig. 3(a) we plot the momentum distribution of the probe as a function of $\mathbf{k}-\mathbf{k}_{p b}$. Thus, in the reference frame of the probe, the OPO signal has a definite homogeneous current [straight arrow in Figs. 3(b) and 3(f)], while the vortex (antivortex) probe has anticlockwise (clockwise) winding constant currents. Figures 3(b) and 3(f) show the real space emission of the signal 4 ps after the $m=1$ $(m=-1)$ LG probe arrival. Images are taken by subtracting the steady state OPO. Also, as in Ref. [9], in our experiments, the gain triggered by the probe in the signal (and lasting around $25 \mathrm{ps}$ ) hinders the observation of the underlying signal dynamics, so that in Fig. 3 we show the signal evolution after the decay of this extra population. According to the previous analysis of Fig. 1, we can therefore predict the location of the "unintended" antivortex (vortex) in Fig. 3(c) [Fig. 3(g)], namely, where the signal and probe currents are antiparallel. In particular, in Fig. 3(c) the antivortex appears on the opposite side of the vortex in Fig. 3(g). Despite the many-shot average, the dynamics of V-AV pairs can be experimentally followed for about 30 ps [Figs. 3(c)-3(e) and 3(g)-3(i)], thereafter the pair eventually annihilates.

Finally, we show that it is possible to create a V-AV pair with just a Gaussian probe, when there is a difference in the signal and probe currents. To this end, we shine a Gaussian pulsed beam either at rest with respect to the OPO signal, $\mathbf{k}_{p b}=\mathbf{k}_{s} \simeq 0$, or moving $\mathbf{k}_{p b} \neq \mathbf{k}_{s}$. No pair appears in the first case, while in the second, a V-AV pair appears on opposite sides of the probe edge-see Fig. 4. Note that circulation is as expected, anticlockwise (clockwise) for the vortex (antivortex) on the upper (lower) side of the probe.

To conclude, the mechanism for $\mathrm{V}-\mathrm{AV}$ pair formation reported here differs from the $\mathrm{V}-\mathrm{AV}$ binding-unbinding associated to the Berezinskii-Kosterlitz-Thouless phase transition, recently adopted to interpret the V-AV observation in nonresonantly pumped polaritons [11]. In our case, the pair onset can be explained in terms of the OPO and probe relative currents, a simple mechanism which does not require resorting to phase fluctuations induced by the pump.

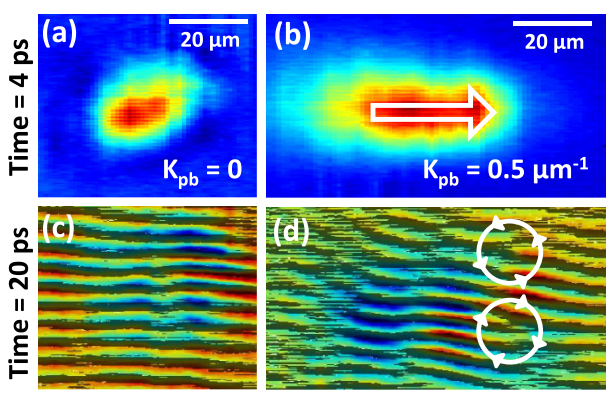

FIG. 4 (color online). Gaussian probe at rest $\mathbf{k}_{p b}=0$ (a) and moving $\mathbf{k}_{p b} \neq 0$ (b) shined on the vortex-free OPO signal. The measured emission shows that no V-AV pairs are created if the probe is at rest (c), while a pair appears after about $15 \mathrm{ps}$ for a moving probe $(\mathrm{d})$.

G. T. acknowledges financial support from FPI scholarship and F. M. M. from the Ramón y Cajal program. Work was supported by the Spanish MEC (MAT2008-01555, QOIT-CSD2006-00019), CAM (S-2009/ESP-1503) and FP7 ITN Clermont4 (235114).

*francesca.marchetti@uam.es

${ }^{\dagger}$ Also at London Centre for Nanotechnology, UK.

[1] L. Onsager, Nuovo Cimento 6, 279 (1949).

[2] R. Feynman, of Progress in Low Temperature Physics (Elsevier, New York, 1955), Vol. 1, pp. 17-53.

[3] J. F. Nye and M. V. Berry, Proc. R. Soc. A 336, 165 (1974).

[4] K. Staliunas and V. J. Sanchez-Morcillo, Transverse Patterns in Nonlinear Optical Resonators (SpringerVerlag, Berlin, 2003).

[5] A. S. Desyatnikova, Y. S. Kivshara, and L. Torner, Prog. Opt. 47, 291 (2005).

[6] K. G. Lagoudakis et al., Nature Phys. 4, 706 (2008).

[7] K. G. Lagoudakis et al., Science 326, 974 (2009).

[8] K. G. Lagoudakis et al., Phys. Rev. Lett. 106, 115301 (2011).

[9] D. Sanvitto et al., Nature Phys. 6, 527 (2010).

[10] D. N. Krizhanovskii et al., Phys. Rev. Lett. 104, 126402 (2010).

[11] G. Roumpos et al., Nature Phys. 7, 129 (2011).

[12] G. Nardin et al., Nature Phys., doi: 10.1038/nphys1959 (2011).

[13] D. Sanvitto et al., arXiv:1103.4885.

[14] M. Wouters and V. Savona, Phys. Rev. B 81, 054508 (2010).

[15] F. M. Marchetti, M. H. Szymańska, C. Tejedor, and D. M. Whittaker, Phys. Rev. Lett. 105, 063902 (2010).

[16] M. H. Szymańska, F. M. Marchetti, and D. Sanvitto, Phys. Rev. Lett. 105, 236402 (2010).

[17] A. V. Gorbach, R. Hartley, and D. V. Skryabin, Phys. Rev. Lett. 104, 213903 (2010).

[18] R. M. Stevenson et al., Phys. Rev. Lett. 85, 3680 (2000).

[19] J. J. Baumberg et al., Phys. Rev. B 62, R16247 (2000).

[20] A. Amo et al., Nature (London) 457, 291 (2009).

[21] D. M. Whittaker, Phys. Status Solidi C 2, 733 (2005).

[22] See Ref. [15] for details and choice of parameters.

[23] M. Perrin, P. Senellart, A. Lemaître, and J. Bloch, Phys. Rev. B 72, 075340 (2005). 